\title{
How sedentary and physically active are breast cancer survivors, and which population subgroups have higher or lower levels of these behaviors?
}

Terry Boyle ${ }^{1,2,3}$, Jeff K. Vallance ${ }^{4}$, Emily K. Ransom³ ${ }^{3}$ Brigid M. Lynch ${ }^{5,6,7}$

1. Cancer Control Research, British Columbia Cancer Agency, Vancouver, British Columbia, Canada

2. School of Population and Public Health, The University of British Columbia, Vancouver, British Columbia, Canada

3. Centre for Medical Research, The University of Western Australia, Perth, Western Australia, Australia

4. Faculty of Health Disciplines, Athabasca University, Athabasca, Canada

5. Cancer Epidemiology Centre, Cancer Council Victoria, Melbourne, Australia

6. School of Population and Global Health, The University of Melbourne, Melbourne, Australia

7. Physical Activity Laboratory, Baker IDI Heart and Diabetes Institute, Melbourne, Australia

\section{Corresponding Author}

\author{
Terry Boyle \\ Cancer Control Research \\ BC Cancer Agency \\ 675 West 10th Avenue \\ Vancouver BC V5Z 1L3 CANADA \\ Tel: (+ 1) 6046758066 \\ Faax: (+1) 6046758180 \\ E-mail: tboyle@bccrc.ca
}

Word Count: 3369

Tables: 3

Figures: 1 


\begin{abstract}
Purpose: Physical activity (PA) and sedentary behavior may influence the physical and mental health of breast cancer survivors, however few studies have objectively measured these behaviors in this population. We used accelerometers to measure the PA and sedentary time levels of breast cancer survivors, and examined the demographic, behavioral and medical correlates of these behaviors using two complementary approaches.
\end{abstract}

Methods: A total of 259 breast cancer survivors wore an accelerometer for seven days during waking hours, and completed a questionnaire. We used linear regression and classification trees to investigate correlates of PA and sedentary time.

Results: The breast cancer survivors in this study (mean age=61 years; mean time since diagnosis=three years) were sedentary for a daily average of 8.2 hours, in light-intensity PA for 5.8 hours and in moderate-to-vigorous intensity PA (MVPA) for 32 minutes, with 16\% meeting PA guidelines. Participants with high comorbidity were the least likely to be meeting guidelines (0\%), while a subgroup of participants with no/low comorbidity, a university degree and higher levels of pre-diagnosis MVPA were the most likely to be meeting guidelines (47\%). Older participants (70+ years) were the most likely to have sedentary time levels at least twice as high as activity levels, while participants who were younger than 70 years and not in the lowest category of pre-diagnosis MVPA were the least likely.

Conclusions: Interventions to facilitate physical activity and reduce sedentary time among breast cancer survivors should consider comorbidity status and previous PA experience.

Keywords: cancer; oncology; survivorship; breast cancer; physical activity; sedentary behavior 


\section{INTRODUCTION}

Physical activity after a breast cancer diagnosis has positive effects on fitness, body size, fatigue and quality of life [1], and recent evidence from observational studies suggests that physical activity may also be associated with increased survival and reduced risk of recurrence [2]. Emerging evidence also indicates that sedentary behavior has adverse health consequences, although there is limited research on this topic among breast cancer survivors [3]. Previous research indicates that between $22 \%$ and $54 \%$ of breast cancer survivors meet physical activity guidelines [4-10]. However, most of the studies conducted to date have been limited by use of self-report measures, which show poor to fair absolute agreement with objective measures of physical activity and sedentary time in cancer survivors [11]. The limited research concerning sedentary time suggests that breast cancer survivors are sedentary for nine to eleven hours per day $[9,10,12]$.

Previous research has identified several potential correlates of physical activity and/or sedentary time in breast cancer survivors, including ethnicity, obesity, smoking status, prediagnosis physical activity levels, fatigue, depression, and cancer treatment [4-7, 9, 13, 14]. However, much of this research has been limited by the use of self-reported measures of physical activity. In addition, previous studies have only used regression modelling to identify individual factors that are associated with higher or lower levels of physical activity and/or sedentary time. While this method provides important and useful information, it is limited in its ability to test for interaction between individual correlates, and often results in difficult to interpret interaction effects when a variable has more than two categories and/or multiple interactions are present [15]. Classification tree-based analyses are able to consider any number of combinations of correlates to identify mutually exclusive subgroups that optimally predict the dependent variable. 
Classification tree-based analyses have been used in previous studies to identify correlates of various health behaviors, such as physical activity [16, 17] and cancer screening adherence [18]. Using classification trees to identify specific population subgroups with lower physical activity and/or higher sedentary time levels, in addition to using regression-based analyses to identify individual variables that are correlated with these behaviors, will provide a more complete picture of sedentary time and physical activity predictors and allow for better targeted interventions.

The aims of this study were to: (1) objectively measure and describe in detail the sedentary time, light-intensity physical activity (LPA) and moderate-to-vigorous physical activity (MVPA) levels of breast cancer survivors; (2) identify the demographic, behavioral and medical correlates of these behaviors; and (3) use classification-tree based analyses to determine specific population subgroups with higher or lower levels of these behaviors.

\section{METHODOLOGY}

The Accurate Measurement of Physical Activity and Sedentary Time among Breast Cancer Survivors (ACCEL-Breast) study was a cross-sectional study conducted in Western Australia (WA) in 2013 [19]. Breast cancer survivors invited to participate in this study had previously taken part in a case-control study of breast cancer that was conducted between 2009 and 2011 [20]. Eligibility criteria for the current study included being female, aged 18 to 80 years of age at the time of diagnosis, being one to three years post-diagnosis, and residing in WA at the time of diagnosis. Breast cancer survivors who had been diagnosed with a subsequent cancer or were currently receiving chemotherapy or radiotherapy were ineligible - this information was obtained via self-report from the participants after they had been invited to take part in the study. Written informed consent was obtained from all participants, and the study was 
approved by Human Research Ethics Committees at the WA Department of Health and The University of WA.

A total of 600 breast cancer survivors were sent a study invitation letter, study information sheet, consent form and a reply-paid envelope. A reminder letter was sent to nonresponders approximately one month after the initial invitation. Breast cancer survivors who agreed to take part in the study were then sent the study questionnaire and an accelerometer (with instructions), which they were instructed to wear on an elasticised waistband over their right hip during waking hours for seven days.

\section{Accelerometer data}

Physical activity and sedentary time were derived from Actigraph GT3X-Plus accelerometers (Actigraph, LLC, Pensacola, FL). The accelerometer data were processed using 60-second epochs. Commonly accepted activity count cut-offs were used to differentiate sedentary time $[<100$ counts-per-minute (CPM)], LPA (100-1,951 CPM) and MVPA ( $\geq 1952$ CPM) [21, 22]. Non-wear time was defined as intervals of at least 60 consecutive minutes of zero counts, with allowance for up to two minutes of observations of less than 50 counts per minute within the non-wear interval. To be considered valid, days of data collection required at least 600 minutes of wear time and no excessive counts (>20,000 counts per minute) [22].

We also derived time spent in sedentary bouts of 20 or more consecutive minutes; this duration of time has been shown to adversely affect cardio-metabolic biomarkers [23]. Finally, we considered time spent in MVPA bouts of ten or more consecutive minutes, and classified participants as meeting or not meeting physical activity guidelines (most physical activity guidelines recommend that MVPA be performed in bouts of ten or more minutes) [24, 25]. Participants who did 150 or more minutes of MVPA per week (with vigorous-intensity physical 
activity double-weighted [25]) were considered to be meeting physical activity guidelines.

Weekly minutes were calculated by multiplying the average daily amount by seven.

\section{Case-control, questionnaire and cancer-related data}

The following variables were obtained from the case-control study: socioeconomic status at diagnosis, smoking status at diagnosis, number of children at diagnosis, body mass index at diagnosis, average hours per week of MVPA in the year before diagnosis, usual sleep quality, menopausal status at diagnosis, dog ownership at diagnosis, and morning/eveningness. Socioeconomic status was based on residential postcode and was derived from the Index of Relative Socio-Economic Disadvantage from the Socio-Economic Indexes for Areas [26]. All other variables were obtained via self-report. MVPA in the year before diagnosis was derived from a self-reported physical activity questionnaire that collected information about lifetime recreational, household and transport-related physical activity [27, 28]. Morning/eveningness was assessed using the Horne-Ostberg scale and participants were classified as evening types, morning types or neither [29].

The following variables were obtained via a self-administered questionnaire in the ACCEL-Breast study: marital status, current employment status, educational attainment, hormone therapy status, breast cancer treatment, and comorbidity. Participants were also asked if a doctor had ever given them a prescription for exercise (i.e., told them specifically what exercise to do). Time since diagnosis, breast cancer stage at diagnosis and breast cancer grade at diagnosis were derived from data obtained from the Western Australian Cancer Registry. Time since diagnosis, age-group, socioeconomic status and breast cancer grade were available for both participants and non-participants. 


\section{Analysis}

We used chi-square tests to determine if the distribution of age-group, socioeconomic status, breast cancer grade and/or time since diagnosis differed between the participants and nonparticipants. Descriptive statistics were used to characterize the demographic, behavioral and medical characteristics of the participants, as well as the sedentary time and physical activity variables. We used linear regression models to investigate the associations between demographic, behavioral and medical correlates and daily minutes of sedentary time, LPA and MVPA, with all analyses adjusted for average daily minutes of accelerometer wear-time. Correlates with a p-value of less than 0.1 in the univariate analyses were then included in the final multivariate model.

Finally, we performed two chi-square automated interaction detection (CHAID) analyses [30]. CHAID is a procedure that uses chi-square statistics to grow a classification (decision) tree. CHAID was used to optimally predict population subgroups that were: (1) more (or less) likely to meet physical activity guidelines; and (2) more (or less) likely to have sedentary time levels at least twice as high as their activity levels (LPA and MVPA combined). Only variables that were included in the final model for daily minutes of MVPA were included for consideration in the meeting MVPA guidelines CHAID analysis, and only variables that were included for the final model for daily minutes of sedentary time and/or LPA were included for consideration in the sedentary-activity ratio CHAID analysis. To ensure the population subgroups identified by the CHAID procedure were of a meaningful size, a minimum of 52 participants (20\% of the sample) was required for a variable to split and a minimum of 26 participants (10\% of the sample) was required in each terminal node. At each level of the classification tree, categories of a variable could be automatically merged to optimally predict the dependent variable. 
A two-sided $P$-value of $<0.05$ was considered statically significant, unless otherwise indicated. Stata 13.1 (StataCorp, College Station TX) was used for all analyses.

\section{RESULTS}

\section{Participants}

A total of 600 breast cancer survivors were invited to take part in this study, 48 of whom were deemed to be ineligible as they were either currently receiving chemotherapy or radiotherapy for their breast cancer or they had been diagnosed with another cancer. Of the 552 eligible survivors, 340 (61.6\%) agreed to take part in the study, and 274 (49.6\%) went on to complete the study. There were no meaningful or statistically significant differences between the participants and non-participants for age, area-level socioeconomic status, time since diagnosis or cancer grade (as shown in Table 1 in Online Resource 1).

Valid accelerometer data were obtained from 267 participants, 259 of whom also completed the questionnaire. The demographic, behavioral and medical characteristics of these 259 participants are displayed in Table 1. Participants were between 36 and 84 years of age (median age $=61$ years) and were 2.3 to 3.7 years post-diagnosis (median time since diagnosis $=$ 3.0 years). Only $15.4 \%$ of the participants could recall being told specifically what exercise to do by a doctor.

\section{Accelerometer-assessed physical activity and sedentary time}

Participants wore the accelerometer for an average of 14.5 hours per day (median $=14.6$

hours) (Table 2). Participants were sedentary for an average of 8.2 hours per day (median $=8.2$ hours), and had a daily average of 5.8 hours of LPA (median = 5.7 hours), 32 minutes of moderate-intensity physical activity (median = 27 minutes), and one minute of vigorous-intensity 
physical activity (median $=0.2$ minutes). Overall, participants spent an average of $56.4 \%$ of their accelerometer-wear hours sedentary, 39.8\% in light-intensity physical activity, and 3.6\% in MVPA.

Approximately 13\% of participants had sedentary time levels that were at least twice as high as their activity levels (LPA and MVPA combined), and only $14.7 \%$ of the participants were meeting physical activity guidelines (performing 150 minutes of MVPA per week in bouts of 10 or more minutes).

\section{Correlates of physical activity and sedentary time}

\section{Linear regression analyses}

All variables that were associated with one or more of sedentary time, LPA and MVPA at the significance level of 0.10 in univariate analyse are shown in Table 2 . In multivariate analyses, age was significantly associated with sedentary time; participants aged 50 to 59 years had significantly lower sedentary time levels than participants aged 30 to 49 years and participants aged 70 to 84 years. Participants with a trade or technical qualification were sedentary for 35.7 more minutes per day $(95 \%$ CI $=7.4,64.0)$ and in LPA for 31.6 fewer minutes per day $(95 \% \mathrm{CI}=-57.9,-5.2)$ than participants who did not complete high school. Participants with the highest level of pre-diagnosis MVPA had significantly lower levels of sedentary time than those with the lowest level of pre-diagnosis MVPA (-46.4 minutes, 95\% CI = -73.1, -19.7), as well as significantly higher levels of LPA (31.9 minutes, 95\% CI = 6.9, 56.8) and current MVPA (12.4 minutes, 95\% CI = 4.8, 20.1). Participants who were morning types had significantly higher levels of MVPA than evening types (10.5 minutes, 95\% CI = 2.3, 18.7), while having two children (compared with having no children) and having a family history of breast cancer were also associated with significantly higher levels of MVPA. 


\section{Classification Tree Analysis}

Four population subgroups were identified in the classification tree analysis for meeting physical activity guidelines (Figure 1). The subgroup least likely to be meeting physical activity guidelines consisted of participants with the highest levels of comorbidity; none of the 39 participants in this group were meeting guidelines. The participants in the subgroup most likely to be meeting guidelines had no or low comorbdity, a university degree, and reported performing more than six hours per week of pre-diagnosis MVPA; $47 \%$ of the participants in this subgroup were meeting physical activity guidelines.

Three population subgroups were identified in the sedentary time-activity ratio classification tree analysis. A subgroup consisting of participants aged 70 years and above was the most likely to have sedentary time levels at least twice as high as their activity levels (29\%). Among those aged younger than 70 years, participants who reported 2.5 or more hours of prediagnosis MVPA were less likely than those who reported fewer than 2.5 hours of pre-diagnosis MVPA to have sedentary time levels at least twice as high as their activity levels (6\% compared with 19\%).

\section{DISCUSSION}

In this study we found that, on average, breast cancer survivors were sedentary for $57 \%$ of their waking hours, in LPA for 40\% of their waking hours, and in MVPA for the remainder (around 32 minutes per day). Factors associated with accelerometer-assessed sedentary time and/or activity levels in regression analyses included pre-diagnosis MVPA levels (sedentary time, LPA and MVPA), education (sedentary time and LPA), age (sedentary time), number of children (MVPA), morning/eveningness (MVPA), and family history of breast cancer (MVPA). In classification tree analyses we found that participants with higher comorbidity were the least 
likely to be meeting physical activity guidelines, while a subgroup consisting of participants with no or low comorbidity, a university degree and higher levels of pre-diagnosis MVPA were the most likely to be meeting physical activity guidelines. Older participants (70 years and older) were the most likely to have sedentary time levels at least twice as high as activity levels. Participants who were younger than 70 years and not in the lowest category of pre-diagnosis MVPA were the least likely to have sedentary time levels at least twice as high as activity levels.

We found that only $15 \%$ of the breast cancer survivors in this study were meeting physical activity guidelines. Two previous studies that have used an objective measure of physical activity have reported higher proportions of breast cancer survivors meeting guidelines (22\% and 43\%) [9, 10], however both of these studies used total accumulated MVPA rather than only considering MVPA bouts as was done in the present study. Not surprisingly, given that people overestimate their physical activity levels on self-reported questionnaire [11], studies that have used self-report measures of physical activity generally report higher proportions of breast cancer survivors meeting physical activity guidelines, with estimates ranging from $34 \%$ to $54 \%$ [4-8].

Breast cancer survivors in this study were sedentary for an average of 8.2 hours per day. This figure differs to results from the three previous studies that have objectively measured sedentary time in breast cancer survivors: Sabiston et al. found that participants were sedentary for an average of 10.8 hours per day (79\% of accelerometer wear-time) [9], while Lynch et al. and Phillips et al. found that breast cancer survivors were sedentary for just over nine hours per day (66\% of wear-time)) [10, 12]. Differences in accelerometer wear-time, methodological differences (e.g., different accelerometer models and/or different processing rules for the accelerometer data) and different participant characteristics across these studies are likely to have 
contributed to the varying results. Regardless, the limited research to date suggests that breast cancer survivors are spending $57 \%$ to $79 \%$ of their waking hours sedentary, with little known about the health effects of sedentary time in this population.

In this study we used two complementary methods analyses to investigate the correlates of sedentary time and physical activity levels: linear regression analyses to investigate the influence of individual correlates, and classification tree analyses to identify population subgroups with higher or lower levels of these behaviors. In the regression analyses higher prediagnosis MVPA levels were associated with significantly higher post-diagnosis levels of MVPA and LPA and significantly lower levels of sedentary time in this study. Higher levels of prediagnosis physical activity levels have been consistently associated with post-diagnosis physical activity levels in breast cancer survivors $[4,14]$. We also found that breast cancer survivors who were morning types performed ten more minutes of MVPA per day than those who were evening types. Although there is limited research concerning the influence that chronotype may have on physical activity and sedentary time levels, data from the Nurses’ Health Study II [31], a study conducted among female working professionals [32] and several studies conducted among adolescents and university students [33-37] all indicate that morning people are more physically active than evening people, and suggest that chronotype may be a novel predictor of physical activity levels. Number of children was also associated with MVPA levels in this study. Previous research conducted among the general population provides mixed results on this topic, with some studies finding that middle-aged women with children at home have lower levels of physical activity than women without children, and other studies finding the opposite [38, 39]. Given the age of the participants in this study, one possibility for our finding is that women with more children may have had more grandchildren, and caring for and playing with these grandchildren 
may have contributed to their MVPA levels. However this is purely speculative, as we did not collect information about the age of the participants' children or the number of grandchildren (or how much time each week the participants spent with their grandchildren). Education (sedentary time and LIPA), age (sedentary time) and family history of breast cancer (MVPA) were also associated with sedentary and activity levels in the current study.

While the above findings from the regression analyses provide important information about individual correlates, they do not take into account possible interactions. In classification tree analyses we found that a subgroups of participants with no or low comorbidity, a university degree and higher levels of pre-diagnosis MVPA were the most likely to be meeting physical activity guidelines, with nearly half of the participants in this subgroup meeting guidelines. Conversely, none of the participants who reported higher levels of comorbidity were meeting physical activity guidelines. Older participants (70 years and older) were the most likely to have sedentary time levels at least twice as high as activity levels, while a subgroup of participants who were younger than 70 years and not in the lowest category of pre-diagnosis MVPA were the least likely to have sedentary time levels at least twice as high as activity levels. Although the findings from the classification tree analyses need to be replicated, they provide some information about where interventions to increase activity levels and decrease sedentary time levels among breast cancer survivors may be best directed.

This study has several strengths and limitations that should be taken into account. A major strength of this study was the use of accelerometers to measure physical activity and sedentary time. Further strengths were our use of two complementary methods (regression and classification tree analyses) to obtain a more complete picture of the correlates of sedentary time and physical activity, and our ability to examine the associations between these behaviors and a 
wide range of pre-diagnosis and/or post-diagnosis demographic, medical and behavioral variables. The study response rate (50\%) raises the possibility that the recruited sample is not representative of all those who were eligible to participate, however we did not observe any meaningful demographic or clinical differences between the participants and non-participants. Finally, the 'counts per minute' approach used to identify sedentary time in this study means that were not able to distinguish between 'stationary standing' and sitting, so the estimates of daily sedentary time estimates may be inflated.

This study was one of the first to objectively assess the activity and sedentary time levels of breast cancer survivors. We found breast cancer survivors were sedentary for $57 \%$ of their waking hours, and only 15\% were meeting physical activity guidelines. Further, unique demographic, medical, and behavioral variables were associated with MVPA and sedentary time. These findings can be used to inform, develop, and evaluate targeted physical activity and sedentary behavior interventions for breast cancer survivors.

\section{ACKNOWLEDGEMENTS}

The ACCEL-Breast study was funded by a Project Grant from the Breast Cancer Research Centre - Western Australia. The case-control study (the Breast Cancer Environment and Employment Study) was funded by the Australian National Health and Medical Research Council (\#573530). Terry Boyle is supported by an Early Career Fellowship from the Australian National Health and Medical Research Council (\#1072266), a Fellowship from the Canadian Institutes of Health Research (\#300068), a Postdoctoral Fellowship from the Michael Smith 
Foundation for Health Research (\#5553), and an Honorary Killam Postdoctoral Research Fellowship from Killam Trusts/The University of British Columbia. Jeff Vallance is supported by the Canada Research Chairs program and a Population Health Investigator Award from Alberta Innovates - Health Solutions. Brigid Lynch is supported by an Early Career Fellowship from the National Breast Cancer Foundation. We would like to acknowledge the Chief Investigators of the case-control study (in particular Professor Lin Fritschi from the School of Public Health at Curtin University and Professor Jane Heyworth from the School of Population Health at The University of Western Australia), and Ms Jessica Occleston at the Baker IDI Heart and Diabetes Institute for her assistance in data processing. We also sincerely thank the people who took the time to participate in this study.

\section{CONFLICT OF INTEREST}

The authors declare they have no conflict of interest.

\section{ETHICAL APPROVAL}

All procedures performed in studies involving human participants were in accordance with the ethical standards of the institutional and/or national research committee and with the 1964 Helsinki declaration and its later amendments or comparable ethical standards.

\section{INFORMED CONSENT}

Informed consent was obtained from all individual participants included in the study. 


\section{REFERENCES}

1. Schmitz K (2011) Physical Activity and Breast Cancer Survivorship. In: Courneya KS, Friedenreich CM (eds) Physical Activity and Cancer. Springer Berlin Heidelberg, p 189215

2. Schmid D, Leitzmann MF (2014) Association between physical activity and mortality among breast cancer and colorectal cancer survivors: a systematic review and metaanalysis. Ann Oncol 25:1293-1311

3. Lynch BM, Dunstan DW, Vallance JK, Owen N (2013) Don't take cancer sitting down. Cancer 119:1928-1935

4. Mason C, Alfano CM, Smith AW, Wang CY, Neuhouser ML, Duggan C, Bernstein L, Baumgartner KB, Baumgartner RN, Ballard-Barbash R, McTiernan A (2013) Long-term physical activity trends in breast cancer survivors. Cancer Epidemiol Biomarkers Prev 22:1153-1161

5. Brunet J, Amireault S, Chaiton M, Sabiston CM (2014) Identification and prediction of physical activity trajectories in women treated for breast cancer. Ann Epidemiol 24:837842

6. Hair BY, Hayes S, Tse C-K, Bell MB, Olshan AF (2014) Racial differences in physical activity among breast cancer survivors: Implications for breast cancer care. Cancer 120:2174-2182

7. Keegan TM, Shariff-Marco S, Sangaramoorthy M, Koo J, Hertz A, Schupp C, Yang J, John E, Gomez S (2014) Neighborhood influences on recreational physical activity and survival after breast cancer. Cancer Causes Control 25:1295-1308

8. Blanchard CM, Courneya KS, Stein K (2008) Cancer survivors' adherence to lifestyle behavior recommendations and associations with health-related quality of life: results from the American Cancer Society's SCS-II. J Clin Oncol 26:2198-2204

9. Sabiston CM, Brunet J, Vallance JK, Meterissian S (2014) Prospective examination of objectively assessed physical activity and sedentary time after breast cancer treatment: sitting on the crest of the teachable moment. Cancer Epidemiol Biomarkers Prev 23:1324-1330

10. Phillips SM, Awick EA, Conroy DE, Pellegrini CA, Mailey EL, McAuley E (2015) Objectively measured physical activity and sedentary behavior and quality of life 
indicators in survivors of breast cancer. Cancer: Advance online publication. doi: 10.1002/cncr.29620

11. Boyle T, Lynch BM, Courneya KS, Vallance JK (2015) Agreement between accelerometer-assessed and self-reported physical activity and sedentary time in colon cancer survivors. Support Care Cancer 23:1121-1126

12. Lynch BM, Dunstan DW, Healy GN, Winkler E, Eakin E, Owen N (2010) Objectively measured physical activity and sedentary time of breast cancer survivors, and associations with adiposity: findings from NHANES (2003-2006). Cancer Causes Control 21:283-288

13. George SM, Alfano CM, Wilder Smith A, Irwin ML, McTiernan A, Bernstein L, Baumgartner KB, Ballard-Barbash R (2013) Sedentary behavior, health-related quality of life, and fatigue among breast cancer survivors. J Phys Act Health 10:350-358

14. Bock C, Schmidt ME, Vrieling A, Chang-Claude J, Steindorf K (2013) Walking, bicycling, and sports in postmenopausal breast cancer survivors-results from a German patient cohort study. Psychooncology 22:1291-1298

15. Lemon S, Roy J, Clark M, Friedmann P, Rakowski W (2003) Classification and regression tree analysis in public health: Methodological review and comparison with logistic regression. Ann Behav Med 26:172-181

16. Frank LD, Kerr J, Sallis JF, Miles R, Chapman J (2008) A hierarchy of sociodemographic and environmental correlates of walking and obesity. Prev Med 47:172-178

17. Boslaugh SE, Kreuter MW, Nicholson RA, Naleid K (2005) Comparing demographic, health status and psychosocial strategies of audience segmentation to promote physical activity. Health Educ Res 20:430-438

18. Dominick G, Papas M, Rogers M, Rakowski W (2015) Classification tree analysis to examine influences on colorectal cancer screening. Cancer Causes Control 26:443-454

19. Ransom EK, Lynch BM, Vallance JK, Boyle T (2015) Offering personalized health behavior feedback did not increase response rate: a randomized controlled trial. J Clin Epidemiol 68: 1383-1384

20. Fritschi L, Erren TC, Glass DC, Girschik J, Thomson AK, Saunders C, Boyle T, ElZaemey S, Rogers P, Peters S, Slevin T, D'Orsogna A, de Vocht F, Vermeulen R, 
Heyworth JS (2013) The association between different night shiftwork factors and breast cancer: a case-control study. Br J Cancer 109:2472-2480

21. Freedson PS, Melanson E, Sirard J (1998) Calibration of the Computer Science and Applications, Inc. accelerometer. Medicine and Science in Sports and Exercise 30:777781

22. Matthews CE, Chen KY, Freedson PS, Buchowski MS, Beech BM, Pate RR, Troiano RP (2008) Amount of time spent in sedentary behaviors in the United States, 2003-2004. Am J Epidemiol 167:875-881

23. Dunstan DW, Kingwell BA, Larsen R, Healy GN, Cerin E, Hamilton MT, Shaw JE, Bertovic DA, Zimmet PZ, Salmon J, Owen N (2012) Breaking up prolonged sitting reduces postprandial glucose and insulin responses. Diabetes Care 35:976-983

24. Canadian Society for Exercise Physiology (2011) Canadian Physical Activity Guidelines: 2011 Scientific Statements. Canadian Society for Exercise Physiology, Ottawa, Canada

25. Physical Activity Guidelines Advisory Committee (2008) Physical Activity Guidelines Advisory Committee Report, 2008. U.S. Department of Health and Human Services, Washington, DC

26. Australian Bureau of Statistics (2013) Census of Population and Housing: SocioEconomic Indexes for Areas (SEIFA), Australia, 2011. Catalogue Number 2033.0.55.001. Australian Bureau of Statistics, Canberra, Australia

27. Boyle T, Heyworth J, Bull F, Fritschi L (2013) Test-retest reliability of transport-related physical activity performed over the lifetime. J Phys Act Health 10:626-631

28. Chasan-Taber L, Erickson JB, McBride JW, Nasca PC, Chasan-Taber S, Freedson PS (2002) Reproducibility of a self-administered lifetime physical activity questionnaire among female college alumnae. Am J Epidemiol 155:282-291

29. Horne JA, Östberg O (1977) Individual differences in human circadian rhythms. Biol Psychol 5:179-190

30. Kass GV (1980) An exploratory technique for investigating large quantities of categorical data. Journal of the Royal Statistical Society. Series C (Applied Statistics) 29:119-127

31. Ramin C, Devore EE, Pierre-Paul J, Duffy JF, Hankinson SE, Schernhammer ES (2013) Chronotype and breast cancer risk in a cohort of U.S. nurses. Chronobiol Int 30:11811186 
32. Haraszti RÁ, Purebl G, Salavecz G, Poole L, Dockray S, Steptoe A (2014) Morningnesseveningness interferes with perceived health, physical activity, diet and stress levels in working women: A cross-sectional study. Chronobiol Int 31:829-837

33. Urban R, Magyarodi T, Rigo A (2011) Morningness-eveningness, chronotypes and health-impairing behaviors in adolescents. Chronobiol Int 28:238-247

34. Schaal S, Peter M, Randler C (2010) Morningness-eveningness and physical activity in adolescents. International Journal of Sport and Exercise Psychology 8:147-159

35. Roeser K, Obergfell F, Meule A, Vögele C, Schlarb AA, Kübler A (2012) Of larks and hearts - morningness/eveningness, heart rate variability and cardiovascular stress response at different times of day. Physiol Behav 106:151-157

36. Gaina A, Sekine M, Kanayama H, Takashi Y, Hu L, Sengoku K, Kagamimori S (2006) Morning-evening preference: sleep pattern spectrum and lifestyle habits among Japanese junior high school pupils. Chronobiol Int 23:607-621

37. Kauderer S, Randler C (2012) Differences in time use among chronotypes in adolescents. Biological Rhythm Research 44:601-608

38. Brown WJ, Mishra G, Lee C, Bauman A (2000) Leisure Time Physical Activity in Australian Women: Relationship with Well Being and Symptoms. Res Q Exerc Sport 71:206-216

39. Scharff DP, Homan S, Kreuter M, Brennan L (1999) Factors Associated with Physical Activity in Women Across the Life Span: Implications for Program Development. Women Health 29:115-134 
Table 1: Demographic, medical and behavioural characteristics of the participants in a study conducted among breast cancer survivors in Western Australia, 2013

\begin{tabular}{|c|c|}
\hline & $\begin{array}{c}\text { Participants } \\
(\mathbf{n}=259)\end{array}$ \\
\hline & $\%$ \\
\hline \multicolumn{2}{|l|}{ Age group } \\
\hline 30 to 49 years & 17.7 \\
\hline 50 to 59 years & 27.0 \\
\hline 60 to 69 years & 35.1 \\
\hline 70 to 84 years & 20.1 \\
\hline \multicolumn{2}{|l|}{ Socioeconomic status } \\
\hline Group 1 (lowest socioeconomic status) & 20.1 \\
\hline Group 2 & 26.6 \\
\hline Group 3 & 28.2 \\
\hline Group 4 (highest socioeconomic status) & 25.1 \\
\hline \multicolumn{2}{|l|}{ Country of birth } \\
\hline Australia/New Zealand & 64.9 \\
\hline United Kingdom & 23.2 \\
\hline Other & 12.0 \\
\hline \multicolumn{2}{|l|}{ Marital Status } \\
\hline Not married/divorced/widowed & 23.6 \\
\hline Married/De-facto relationship & 76.4 \\
\hline \multicolumn{2}{|l|}{ Number of Children } \\
\hline 0 & 11.6 \\
\hline 1 & 6.2 \\
\hline 2 & 44.8 \\
\hline $3+$ & 37.5 \\
\hline \multicolumn{2}{|l|}{ Highest level of education } \\
\hline Did not complete high school / high school only & 16.2 \\
\hline Completed high school & 22.0 \\
\hline Trade/technical qualification & 34.4 \\
\hline University degree & 27.4 \\
\hline \multicolumn{2}{|l|}{ Current Working Status } \\
\hline Not working & 48.3 \\
\hline Part-time work & 28.2 \\
\hline Full-tine work & 23.6 \\
\hline \multicolumn{2}{|l|}{ Time between diagnosis \& study participation } \\
\hline 2.25 to 2.59 years & 25.9 \\
\hline 2.6 to 2.9 years & 26.3 \\
\hline 3.0 to 3.24 years & 23.9 \\
\hline 3.25 to 3.7 years & 23.9 \\
\hline \multicolumn{2}{|l|}{ Stage of breast cancer at diagnosis } \\
\hline I & 46.3 \\
\hline II & 30.1 \\
\hline III & 6.9 \\
\hline IV & 5.4 \\
\hline Unknown & 11.2 \\
\hline
\end{tabular}




\begin{tabular}{|c|c|}
\hline \multicolumn{2}{|l|}{ Breast cancer treatment } \\
\hline Surgery only & 20.2 \\
\hline Chemotherapy, no radiotherapy & 14.0 \\
\hline Radiotherapy, no chemotherapy & 30.2 \\
\hline Chemotherapy and radiotherapy & 35.7 \\
\hline \multicolumn{2}{|l|}{ Currently receiving hormone therapy } \\
\hline No & 29.1 \\
\hline Yes & 70.9 \\
\hline \multicolumn{2}{|l|}{ Comorbidity } \\
\hline None & 54.7 \\
\hline Only high blood pressure \&/or high cholesterol & 30.2 \\
\hline Angina, heart attack, stroke \&/or diabetes & 15.1 \\
\hline \multicolumn{2}{|l|}{ Menopausal Status at diagnosis } \\
\hline Premenopausal & 72.6 \\
\hline Postmenopausal & 27.4 \\
\hline \multicolumn{2}{|l|}{ Smoking status at diagnosis } \\
\hline Never & 56.8 \\
\hline Former & 39.8 \\
\hline Current & 3.5 \\
\hline \multicolumn{2}{|l|}{ BMI at diagnosis } \\
\hline Underweight & 1.6 \\
\hline Normal & 48.0 \\
\hline Overweight & 30.9 \\
\hline Obese & 19.5 \\
\hline \multicolumn{2}{|l|}{ MVPA hours/week in year before diagnosis } \\
\hline$<2.5$ hours & 24.3 \\
\hline 2.5 to 5.9 hours & 25.5 \\
\hline 6 to 9.9 hours & 25.1 \\
\hline$\geq 10$ hours & 25.1 \\
\hline \multicolumn{2}{|l|}{ Morningness/Eveningness } \\
\hline Evening person & 16.7 \\
\hline No Preference & 54.9 \\
\hline Morning person & 28.4 \\
\hline \multicolumn{2}{|l|}{ Dog ownership at diagnosis } \\
\hline No & 58.7 \\
\hline Yes & 41.3 \\
\hline \multicolumn{2}{|l|}{ Family history of breast cancer } \\
\hline No & 60.2 \\
\hline Yes & 39.8 \\
\hline
\end{tabular}


Table 2. Accelerometer-assessed physical activity and sedentary time levels of breast cancer survivors $(n=259)$ in Western Australia, 2013

\section{Mean (standard deviation) Median (IQR)}

\begin{tabular}{|c|c|c|}
\hline \multicolumn{3}{|l|}{ Accelerometer wear-time } \\
\hline Minutes per day & $868.4(69.2)$ & $873.4(820.9,917.3)$ \\
\hline \multicolumn{3}{|l|}{ Light-intensity physical activity } \\
\hline Minutes per day & 345.7 (73.5) & $342.6(295.6,390.4)$ \\
\hline \multicolumn{3}{|l|}{ Moderate-intensity physical activity } \\
\hline Minutes per day & $30.6(22.0)$ & $26.0(15.3,41.5)$ \\
\hline \multicolumn{3}{|l|}{ Vigorous-intensity physical activity } \\
\hline Minutes per day & $1.0(2.7)$ & $0.2(0,0.7)$ \\
\hline \multicolumn{3}{|l|}{ Moderate-to-vigorous intensity physical activity } \\
\hline Minutes per day & $31.6(23.1)$ & $26.8(15.2,43.5)$ \\
\hline Minutes per day accumulated in 10 minute bouts & $9.2(12.1)$ & $4.5(0,14.7)$ \\
\hline \multicolumn{3}{|l|}{ Sedentary time } \\
\hline Minutes per day & $489.6(80.9)$ & $492.0(433.9,547.3)$ \\
\hline Minutes per day accumulated in 20 minute bouts & $185.0(83.9)$ & $174.6(124.5,235.4)$ \\
\hline
\end{tabular}


Table 3. Correlates of daily minutes of sedentary time, light-intensity physical activity (LPA) and moderate-to-vigorous intensity physical activity (MVPA) in breast cancer survivors (n=259) in Western Australia, 2013

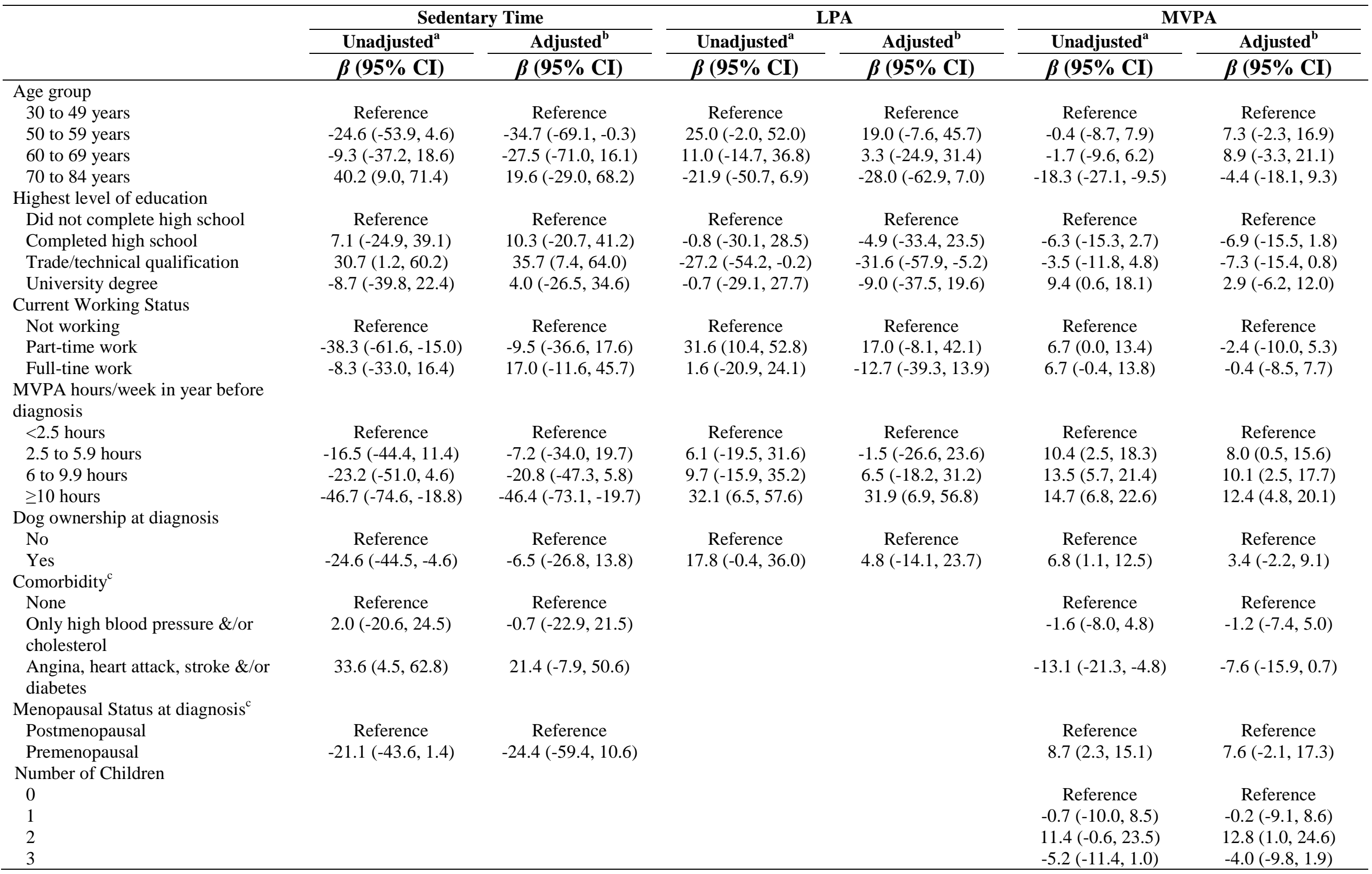




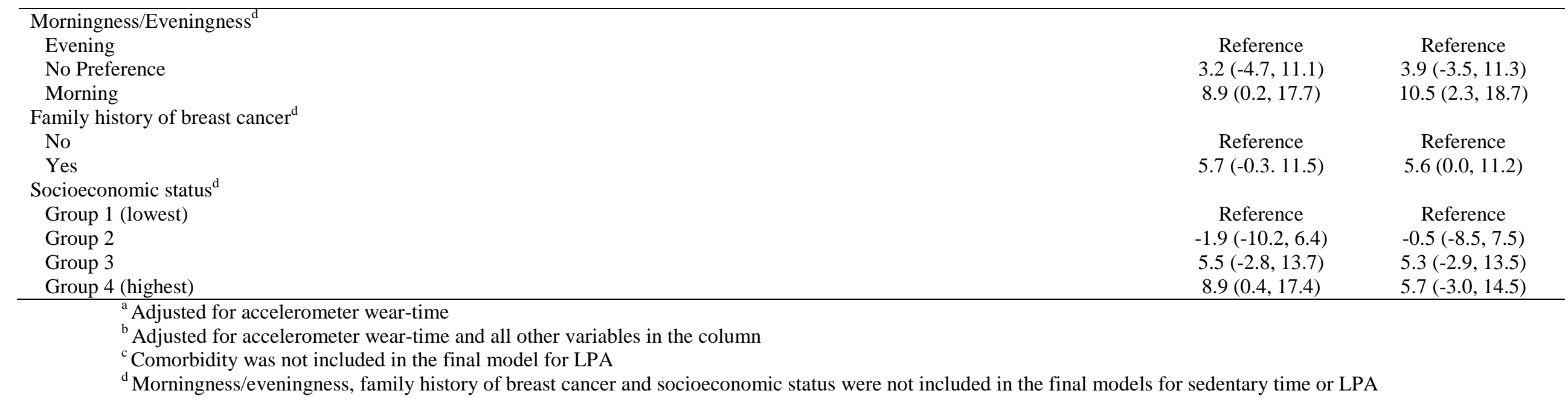




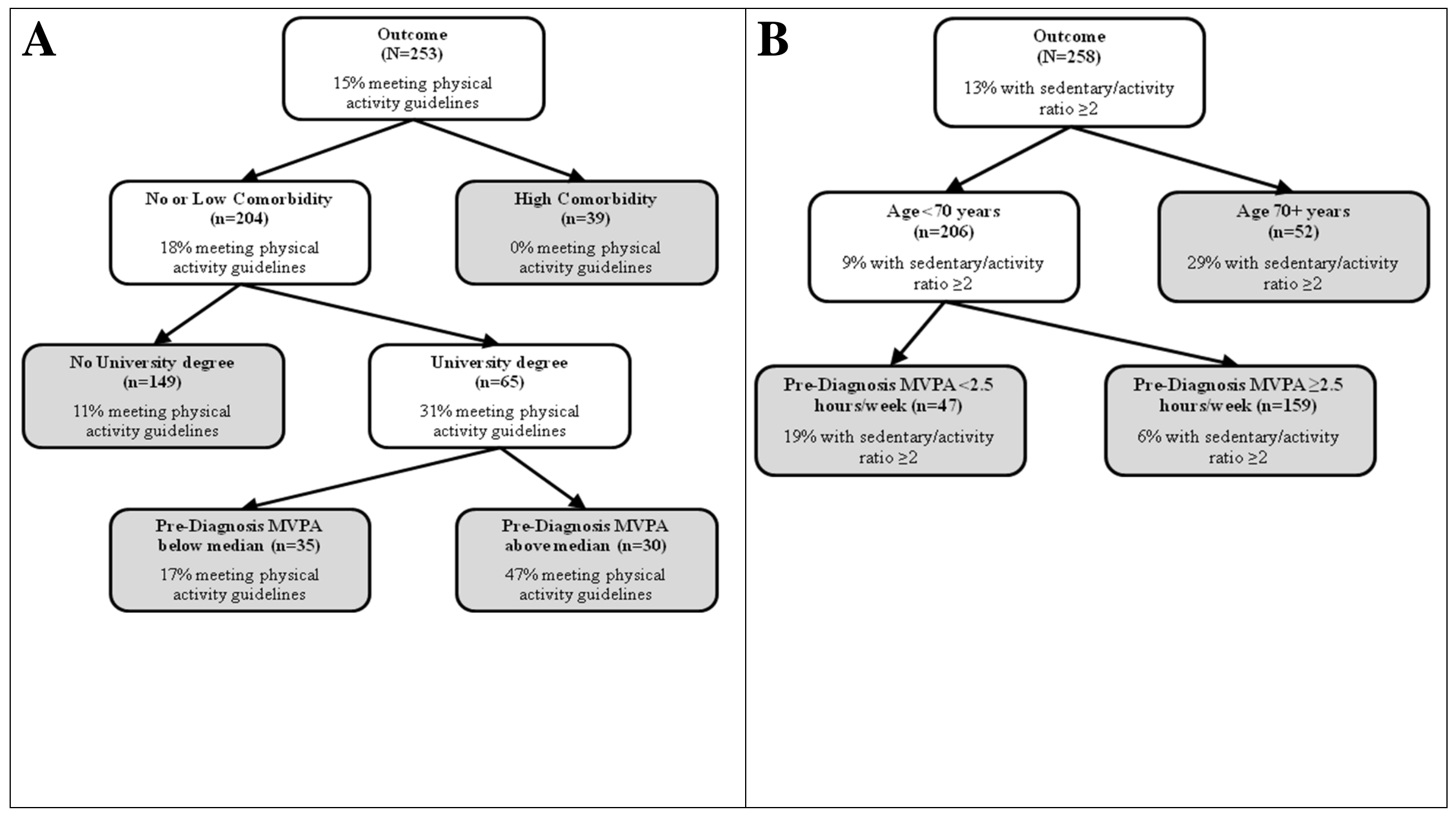

Figure 1: Classification tree analyses that identify population subgroups of breast cancer survivors that are: (A) more (or less)

likely to meet physical activity guidelines; and (B) more (or less) likely to have sedentary time levels at least twice as high as their activity levels 